\title{
Indirect method for quantification of cell biomass during solid-state fermentation of palm kernel cake based on protein content.
}

\begin{abstract}
Solid-State Fermentation (SSF) of Aspergillus niger FTCC 5003 on Palm Kernel Cake (PKC) is a practical approach to upgrade PKC into value added product. Present study was conducted on Aspergillus niger FTCC 5003 growth profile and models that are able to describe the growth in SSF using PKC substrate. Due to the difficulties of separating cell biomass quantitatively from the substrate for SSF systems, indirect method for measurement of cell growth during SSF of PKC by Aspergillus niger FTCC 5003 was studied based on the estimation of glucosamine and protein content. Preliminary relationships between glucosamine and protein contents to fungal dry cell weight (Dw) were developed using simulated homogenous SSF data using glass beads as support materials. Both glucosamine and protein contents were well correlated to the fungal dry cell weight in SSF on support materials for protein and glucosamine, respectively. The equations obtained were used for the estimation of cell biomass profile during SSF of PKC from the data of glucosamine and protein as growth indicator study. The estimated fungal dry cell weight based on protein concentration and $\hat{\mathrm{I}}^{2}$-mannanase activity as metabolic activity for microbial growth were well correlated to PKC dry weight which, indicating that both were suitable marker in describing the growth of A. niger FTCC 5003 in this system. In contrast, estimated fungal dry cell weight based on glucosamine concentration was not suitable to describe the growth of $\mathrm{A}$. niger FTCC 5003.
\end{abstract}

Keyword: Solid state fermentation; Palm kernel cake; Aspergillus niger; Protein; Glucosamine; Dry cell weight. 